\title{
A revision of the Oligodon taeniatus (Günther, 1861) group (Squamata: Colubri- dae), with the description of three new species from the Indochinese Region
}

\author{
PATRICK DAVID ${ }^{1}$, GERNOT VOGEL ${ }^{2} \&$ JOHAN VAN ROOIJEN $^{3}$ \\ ${ }^{1}$ Département Systématique et Evolution, USM 602 Taxonomie-collection-Reptiles \& Amphibiens, Case Postale 30, Muséum \\ National d'Histoire Naturelle, 57 rue Cuvier, F-75231 Paris Cedex 05, France. E-mail: pdavid95@wanadoo.fr \\ ${ }^{2}$ Society for Southeast Asian Herpetology, Im Sand 3, D-69115 Heidelberg, Germany.E-mail: Gernot.Vogel@t-online.de \\ ${ }^{3}$ Tulpentuin 313, 2272 EH Voorburg, The Netherlands. E-mail: j1.van.rooijen@hetnet.nl
}

\section{Table of contents}

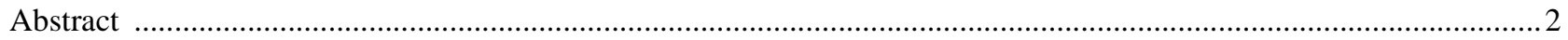

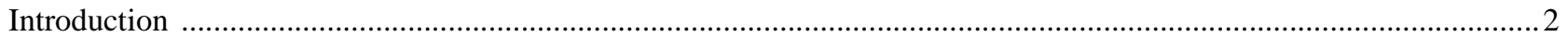

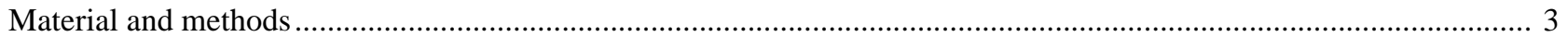

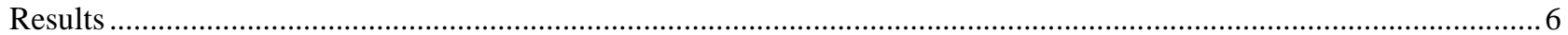

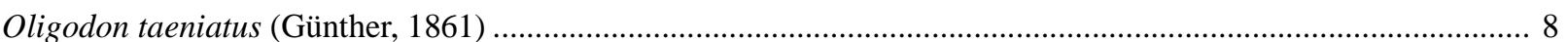

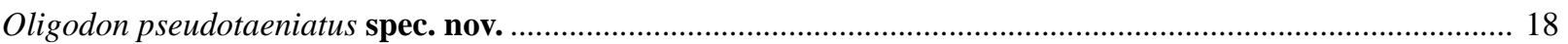

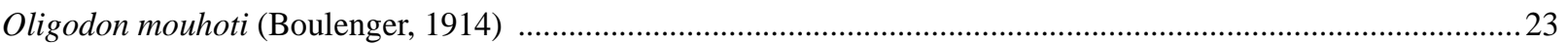

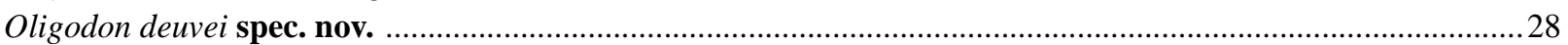

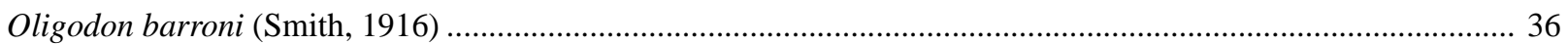

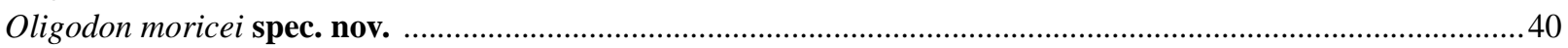

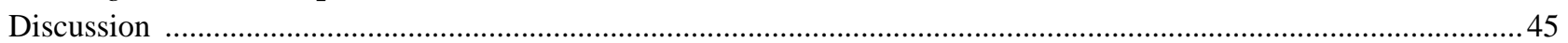

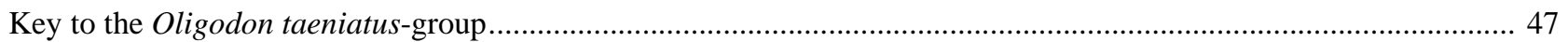

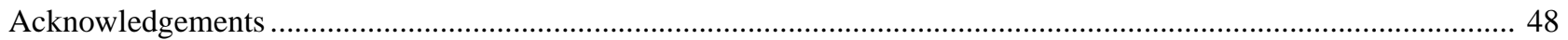

Literature cited 


\begin{abstract}
The group of Asian colubrid species morphologically similar to Oligodon taeniatus (Günther, 1861), previously containing only O. taeniatus, Oligodon mouhoti (Günther, 1864) and Oligodon barroni (Smith, 1916), is revised on the basis of variation in external morphology and dentition of 175 specimens. The confused nomenclatural history of $O$. taeniatus and its name bearing type is discussed. A neotype is described for Simotes quadrilineatus Jan \& Sordelli, 1865, a synonym of $O$. taeniatus. The holotype of Simotes taeniatus var. mouhoti Boulenger, 1914 is identified. Three new species within this group are described. Oligodon pseudotaeniatus spec. nov. is described on the basis of specimens from central Thailand. This species is morphologically similar to Oligodon taeniatus, but differs by the combination of 17 dorsal scale rows at midbody, 8 supralabials, the absence of dark dorsal and tail blotches and the presence of a vertebral stripe edged with black but no dorsolateral stripes. Oligodon deuvei spec. nov. is described on the basis of specimens from southern Vietnam and Laos; it differs from other known species of the group by the combination of 12-15 maxillary teeth, 17 dorsal scale rows at midbody, usually seven supralabials, the absence of dark dorsal and tail blotches and the presence of a broad vertebral stripe, often conspicuously orange or rusty red. This species is most similar to Oligodon barroni but differs from the latter by a higher number of maxillary teeth and the absence of dark dorsal and tail blotches. Lastly, Oligodon moricei spec. nov. is described on the basis of a single specimen from southern Vietnam. It differs from other species by the combination of a broad rusty brown vertebral stripe edged with two broad black stripes, 12 maxillary teeth, 17 dorsal scale rows, a high number of ventral scales, seven supralabials and a dark cloudy or smoky venter. These new species are compared with other species known from the Indo-Chinese Region. The diagnoses of $O$. taeniatus, O. mouhoti and $O$. barroni are revised. A key to members of the group is given.
\end{abstract}

Key words: Oligodon, Oligodon barroni, Oligodon deuvei spec. nov., Oligodon moricei spec. nov., Oligodon mouhoti, Oligodon pseudotaeniatus spec. nov., Oligodon taeniatus, Neotype, Taxonomy, Cambodia, Laos, Thailand, Vietnam

\title{
Introduction
}

The colubrid genus Oligodon Boie, 1827 (type species: Oligodon bitorquatus Boie in Boie, 1827, by monotypy) is widely represented in central and tropical Asia, eastwards as far as eastern Indonesia. As of 1 November, 2007, the genus includes 68 recognized species (The TIGR Reptile Database: http://www.reptiledatabase.org). The taxonomy of this genus is intricate and sorely needs revision. Several species have a limited range and are distinguished from others by a combination of subtle differences in morphological characters, making this genus taxonomically challenging. Nevertheless, Smith (1943) and Wagner (1975, 1976) recognized informal groups within the genus, based largely on hemipenial morphology.

One of these groups constitutes what both Smith (1943) and Wagner (1976) recognized as the "Oligodon taeniatus-group", which includes O. taeniatus (Günther, 1861), Oligodon mouhoti (Günther, 1864), and Oligodon barroni (Smith, 1916). This group is mainly characterized by (1) deeply forked hemipenes with two large papillae (see Smith, 1943: 209), (2) 17 or 19 dorsal scale rows, (3) 10-18 maxillary teeth, a rather high number in the genus Oligodon, (4) a complete head scalation complement, including a loreal and sometimes a presubocular, (5) an entire anal plate; and (6) a striped dorsal pattern, with the presence of one vertebral stripe and, on each side, of one or two dark dorsal stripes. These species are currently known from Thailand (mostly north of the Peninsula), Cambodia, Laos and Vietnam. These small snakes are usually below $450 \mathrm{~mm}$ in total length.

There has been a large amount of confusion in the literature centered on these three morphologically similar taxa. The confusion arose from a mistake made by Günther (1864) on the number of dorsal scale rows of the type of his Simotes taeniatus Günther, 1861, erroneously corrected to 17 rows, whereas the holotype had indeed 19 scale rows as written by Günther (1861). Accepting this unfortunate correction, Jan \& Sordelli (1865) named specimens with 19 rows as Simotes quadrilineatus Jan \& Sordelli, 1865. This confusion led several authors, including Boulenger (1894) and Smith (1943), to use the specific nomen taeniatus for specimens with 17 dorsal scale rows, and quadrilineatus for those with 19 rows. In so doing, Smith (1943) erroneously regarded Simotes taeniatus var. mouhoti Boulenger, 1914 (now Oligodon mouhoti; 17 dorsal scale rows) 